\title{
Morphological Study of Chordae Tendineae of Rightventricle in Embalmed Human Cadavers.
}

\author{
Babita Kujur ${ }^{1}$, Narendra Thakur ${ }^{2}$ and Renu Prasad ${ }^{3}$ \\ ${ }^{I}$ Junior Resident, Department of Anatomy, Rajendra Institute of Medical Sciences, Ranchi \\ ${ }^{2}$ Professor, Department of Anatomy, Rajendra Institute of Medical Sciences, Ranchi \\ ${ }^{3}$ Professor and H.O.D, Department of Anatomy, Rajendra Institute of Medical Sciences, Ranchi
}

\begin{abstract}
:
Introduction: Chordae tendineae are fibrous collagenous structures supporting the leaflets of atrioventricular valve. Chordae tendineae form one of the important components of atrio-ventricular valve complex.They convey the contraction of the papillary muscles to the valve and so prevent the latter's eversion. Dysfunction of these subvalvular components of tricuspid valve isfrequent.

Materials And Methods: The present work was done by dissecting 42 formalin fixed adult human hearts. The sex of human was not considered. The following observations were taken (a) types of chordae tendineae (b)number of chordae tendineae attached to each papillary muscle and their mode oforigin, insertion and distribution.

Observations And Results: In present study, five different types of chordae tendineae were found i.e. rough zone, fan shaped, free edge, deep and basal. The average number of chordae tendineae arising from each papillary muscle were as follows-APM-5.07, PPM- 3.04 and SPM-2.1.The average number of chordae tendineae inserted into each cusp was as follows -anterior cusp-5.64, posterior cusp-4.07, septal cusp -8.64 and at each commissure -0.9.Thus the average number of chordae attached to the tricuspid valve was 19.25.
\end{abstract}

Conclusion: This knowledge may help cardiac surgeons during surgical procedures conducted for correction of valve defects.

Keywords: Human heart, right ventricle, chordae tendineae

\section{Introduction}

Chordae tendineae are fibrous collagenous structures supporting the leaflets of atrioventricular valve. These are of two types - true chordae and false chordae [1]. False chordae connect papillary muscles to each other or to the ventricular wall including the septum, or pass directly between points on the wall (or septum or both).They are irregular in numbers and dimensions in the right ventricle.True chordae usually arise from small projections on the tips or margins of the apical one thirds of papillary muscles, but sometimes arise from bases of the papillary muscles or directly from the ventricular walls and the septum. They are attached to various parts of ventricular aspects or the free margins of the cusps [1].Chordae tendineae were first classified by Tandler into I, II and III order chordae according to the distance of the attachment from the margins of the cusps [2]. This scheme has little functional or morphological merit.Silver et al (1971) depicted five different types of chordae tendineae and named them according to their shapes or their site of insertion on the leaflets - fan shaped, rough zone, basal, free edge and deep [3].

\section{Materials and Methods}

The present work was carried out in department of Anatomy, Rajendra Institute of Medical Sciences (RIMS), Ranchi, Jharkhand. This work was done by dissecting 42 formalin fixed adult human hearts. The sex of human was not considered.

Collection of specimens: The adult human hearts were obtained by dissecting formalin fixed cadavers allotted to the undergraduate medical students of department of anatomy, RIMS, Ranchi, Jharkhand. Few human hearts, used earlier by a senior post graduate student of RIMS, for his dissertation work on coronary arteries were also utilized for the present study. Some of the hearts were also collected from the Department of forensic medicine and toxicology, RIMS, Ranchi.

Method of dissection of already removed hearts: Dissection was performed according to the standard techniques, by opening through theatrioventricular valve to view the constituents of tricuspid valve complex.

Exposure of tricuspid valve: To expose the tricuspid valve, incision was begun at the inferior vena cava with scissors and cut into the right atrium staying about 0.5 to $1 \mathrm{~cm}$ above the tricuspid valve annulus. Blood clots if present were washed out from the atrial chamber. 
Exposure of papillary muscles and chordae tendineae: The incision given earlier for exposure of tricuspid valve was extended further downward vertically along the right border of the right ventricle just above the apex of the heart. Blood clots if present were washed out from the chamber. This exposes the papillary muscles and chordae tendineae of the right ventricle. The chordae tendineae arising from each papillary muscle were identified, their type, number and any variations were noted and tabulated.

\section{Observations and Results}

It was observed that the chordae tendineae were of two types - true and false (photograph no.1). True chordae arose either from a papillary muscle or directly from the ventricular wall and were not only inserted to the corresponding leaflet but also to the adjacent leaflet.It was found that the chordae tendineae arising from the anterior papillary muscle were inserted to both anterior and posterior leaflet of tricuspid valve (photograph no.2), similarly chordae tendineae arising from posterior papillary muscle were inserted to both posterior and septal leaflet. The chordae tendineae arising from septal papillary muscle were inserted to both septal and posterior leaflets.Five different types of chordae tendineae were identified in the wall of right ventricle, namely rough zone (RZC), free edge (FEC), fan shaped (FSC) (photograph no.3), deep chordae (DC) and basal chordae (BC). The average number of chordae attached to each papillary muscle is tabulated below-

Table no.1 - Showing average number of chordae tendineae (CTN) attached to each papillary muscle.

\begin{tabular}{|l|l|l|l|}
\hline & Right ventricle & SPM \\
\hline Papillary muscle & APM & PPM & 2.1 \\
\hline Avg. no. of CTN & 5.07 & 3.04 & \\
\hline
\end{tabular}

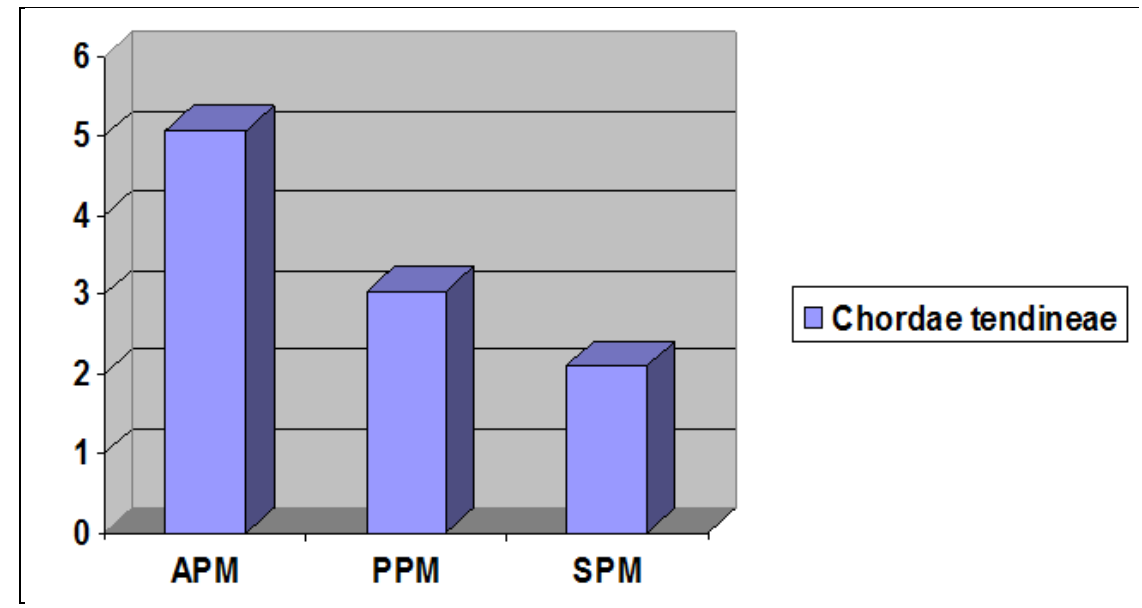

Graph no.1 -Bar graph showing average number of chordae tendineae originating from each papillary muscle. $\mathrm{X}$ axis - Showing different papillary muscles, $\mathrm{Y}$-axis -Showing average number of chordae tendineae

The observation of the insertion of chordae tendineae to different zones of the cusps of right ventricle was done based on the classification given by Silver.

Table no. 2- showing average number of different types of chordae inserted into the leaflets.

\begin{tabular}{|l|l|l|}
\hline Site of insertion & Type of chordae & Average number of chordae inserted in each leaflet \\
\hline \multirow{4}{*}{ Anterior Leaflet } & Fan shaped & 0 \\
\cline { 2 - 3 } & Free edge & 0.61 \\
\cline { 2 - 3 } & Rough & 3.5 \\
\cline { 2 - 3 } & Deep & 1.2 \\
\cline { 2 - 3 } & Basal & 0.5 \\
\hline Posterior Leaflet & Fan shaped & 0.35 \\
\cline { 2 - 3 } & Free edge & 0.76 \\
\cline { 2 - 3 } & Rough & 1.3 \\
\cline { 2 - 3 } & Deep & 1.02 \\
\cline { 2 - 3 } & Basal & 0.57 \\
\hline Septal leaflet & Fan shaped & 0 \\
\cline { 2 - 3 } & Free edge & 1.09 \\
\cline { 2 - 3 } & Rough & 4.8 \\
\cline { 2 - 3 } & Deep & 0.78 \\
\cline { 2 - 3 } & Basal & 1.88 \\
\hline APC & Fan shaped & 0.8 \\
\hline PSC & Fan shaped & 0.5 \\
\hline ASC & Fan shaped & 0.7 \\
\hline
\end{tabular}


Table no.3 -Showing percentage of cases having different types of chordae tendineae attached to each leaflet

\begin{tabular}{|l|l|l|}
\hline Leaflets & Type of chordae & Percentage of cases presenting such chordae in each leaflet \\
\hline Anterior Leaflet & Free edge & 57.14 \\
\cline { 2 - 3 } & Rough & 100 \\
\cline { 2 - 3 } & Deep & 76.19 \\
\cline { 2 - 3 } & Basal & 40.47 \\
\hline Posterior Leaflet & Fan shaped & 33.33 \\
\cline { 2 - 3 } & Free edge & 54.76 \\
\cline { 2 - 3 } & Rough & 90.47 \\
\cline { 2 - 3 } & Deep & 78.57 \\
\cline { 2 - 3 } & Basal & 47.61 \\
\hline Septal leaflet & Fan shaped & 0 \\
\cline { 2 - 3 } & Free edge & 64.28 \\
\cline { 2 - 3 } & Rough & 100 \\
\cline { 2 - 3 } & Deep & 59.52 \\
\cline { 2 - 3 } & Basal & 85.71 \\
\hline
\end{tabular}

The average number of chordae tendineae attached to each cusp of the tricuspid valve is tabulated below-

Table no.4 - Showing average number of chordae tendineae (CTN) attached to each cusp of right ventricle.

\begin{tabular}{|l|l|l|l|l|}
\hline & Anterior cusp & Posterior cusp & Septal cusp & Commissure \\
\hline Avg. no. of CTN & 5.64 & 4.07 & 8.64 & 0.9 \\
\hline
\end{tabular}

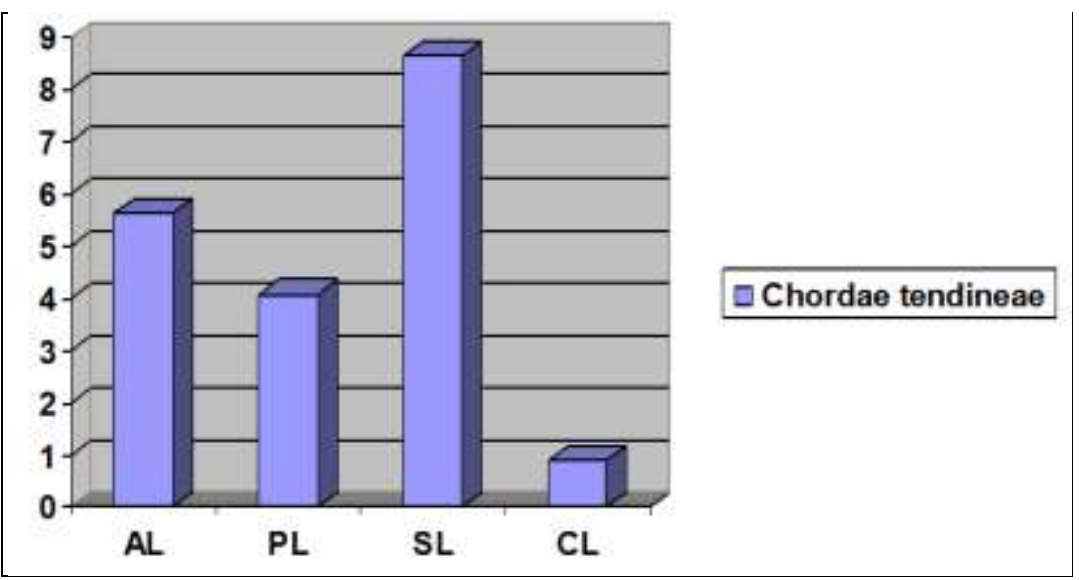

Graph no. 2 - Bar graph showing average number of chordae tendineae attached to each leaflet $\mathrm{X}$-axis Showing leaflet, $\mathrm{Y}$-axis showing average number of chordae.

Thus the average number of chordae tendineae attached to the tricuspid valve was 19.25.

\section{Discussion}

In present study, five different types of chordae tendineae were found i.e. rough zone, fan shaped, free edge, deep and basal. The average number of chordae tendineae arising from each papillary muscle were as follows-APM-5.07, PPM- 3.04 and SPM - 2.1.The average number of chordae tendineae inserted into each cusp was as follows -anterior cusp-5.64, posterior cusp-4.07, septal cusp -8.64 and at each commissure -0.9.Thus the average number of chordae attached to the tricuspid valve was 19.25. According to Nigri et al, 2001 the mean number of cords originating from the APM, PPM and SPM were 4.74, 2.67 and 1.77respectively [4].In 1970 JHC Lam et al studied chordae tendineae in 50 normal mitral valves and classified them according to their mode of insertion into 4 main types. [5]. In the present study involving tricuspid valves 5 types of chordae were identified, namely free edge, fan shaped, rough zone, deep and basal chordae. In1971, Silver MD, Lam HC, Ranganathan N et al studied on the morphology of 50 normal human Tricuspid valve and reported that the surface of the leaflets could be divided into three zones: (1) the rough zone, into which most of the chordae tendineae are inserted, (2) the basal zone, and (3) the clear zone, which lies between the rough and basal zones. [3] Five types of chordae were distinguished by their morphology and mode of insertion: fan-shaped, rough zone, basal, free edge, and deep chordae [3]. The last two types are unique to the tricuspid valve. Fan shaped chordae were present at the anteroposterior commissure in 47 hearts, at the posteroseptal commissure in 50 and at the anteroseptal in 41 hearts. Rough zone chordae were attached to the anterior leaflet in all 50 hearts, to the posterior leaflet in 41 and to the septal leaflet in 49 . The free edge chordae branch before insertion and their fine subdivisions form a delta-shaped insertion at the free edge. One or more were found attached to 32 anterior, 24 posterior, and 25 septal leaflets. Basal chordae were found attached to 23 anterior, 23 posterior, and 45 septal leaflets. On the 
average 25 chordae were inserted into the tricuspid valve. Of the 25 chordae, 7 were inserted to the anterior leaflet, 6 to the posterior leaflet, and 9 to the septal leaflet [3]. According to Skwarek et al 2006, the main leaflets usually received $20.79 \pm 8.43$ cords and the accessory cusps $8.14 \pm 4.85$ cords $[6,10]$. Most of the chordae tendineae arose from the apices but few from thesides of the papillary muscles.Chordae tendineae arising from a papillary muscle were not only inserted to the corresponding leaflet but also to the adjacent leaflet. Similar finding was reported by Mohamed A. B.Motabagani [7].Escande G Guillot et al in1980 described a new type of tendinous cord in the tricuspid valve, which was different from the 4 types described earlier. They named it 'Mixed cord'. The mixed cord is a cylindrical tendinous cord, always ramified into branches. Each ramification is attached to the valve by an expansion, which is either perpendicular or parallel to the ring. [8]In the present study "Mixed cords" were not found.S.A.Gunnal et al defined more than 21 terminologies of chordae tendineae by classifying it into 6 different types [9].

Table no.5: Comparison of average number of CTN attached to different zones of cusps in present study with earlier work.

\begin{tabular}{|l|l|l|l|}
\hline Site of insertion & Types of chordae & Avg. no. of chordae attached in present study & Silver \\
\hline \multirow{5}{*}{ Anterior leaflet } & RZC & 3.5 & 4 \\
\cline { 2 - 4 } & FEC & 0.61 & 1 \\
\cline { 2 - 4 } & DC & 1.2 & 2 \\
\cline { 2 - 4 } & BC & 0.5 & 1 \\
\hline Posterior leaflet & FSC & 0.35 & 1 \\
\cline { 2 - 4 } & RZC & 1.3 & 2 \\
\cline { 2 - 4 } & FEC & 0.76 & 1 \\
\cline { 2 - 4 } & DC & 1.02 & 1 \\
\cline { 2 - 4 } & BC & 0.57 & 1 \\
\hline Septal leaflet & RZC & 4.8 & 1 \\
\cline { 2 - 4 } & FEC & 1.09 & 1 \\
\cline { 2 - 4 } & DC & 0.78 & 3 \\
\cline { 2 - 4 } & BC & 1.88 & 1 \\
\hline APC & FSC & 0.8 & 1 \\
\hline PSC & FSC & 0.5 & 1 \\
\hline ASC & FSC & 0.7 & 1 \\
\hline
\end{tabular}

Table no.6: Comparison of percentage of cases with various types of chordae present in right ventricle in present work with earlier work

\begin{tabular}{|l|l|l|l|}
\hline Leaflet & Type of chordae & \% obtained in present Study & \% obtained by Silver \\
\hline \multirow{5}{*}{ Anterior Leaflet } & RZC & 100 & 100 \\
\cline { 2 - 4 } & FEC & 57.14 & 60 \\
\cline { 2 - 4 } & DC & 76.19 & 70 \\
\cline { 2 - 4 } & BC & 40.47 & 50 \\
\hline Posterior leaflet & FSC & 33.33 & 40 \\
\cline { 2 - 4 } & RZC & 90.47 & 80 \\
\cline { 2 - 4 } & FEC & 54.76 & 50 \\
\cline { 2 - 4 } & DC & 78.57 & 60 \\
\cline { 2 - 4 } & BC & 47.61 & 50 \\
\hline \multirow{5}{*}{ Septal leaflet } & RZC & 100 & 100 \\
\cline { 2 - 4 } & FEC & 64.28 & 50 \\
\cline { 2 - 4 } & DC & 59.52 & 70 \\
\cline { 2 - 4 } & BC & 85.71 & 90 \\
\hline
\end{tabular}

Table no.7: Comparison of average number of chordae tendineae attached to each cusp of tricuspid valve obtained in present study with the earlier work.

\begin{tabular}{|l|l|l|}
\hline Site of insertion & $\begin{array}{l}\text { Avg. no. of CTN attached to each cusp } \\
\text { in present study }\end{array}$ & $\begin{array}{l}\text { Avg. no. of CTN attached to } \\
\text { each cusp described by Silver }\end{array}$ \\
\hline Anterior leaflet & 5.64 & 8 \\
\hline Posterior leaflet & 4.07 & 7 \\
\hline Septal leaflet & 8.64 & 9 \\
\hline Each commissure & 0.9 & 1 \\
\hline
\end{tabular}

\section{Conclusion}

Chordae tendineae forms the important component of the atrioventricular valve complex as it maintains the continuity of the valve complex. The present study shows that there is great variation in number and morphology of chordae. Thus this study may help the cardiac surgeons during surgical procedures conducted for correction of valve defects. 


\section{References}

[1] Susan Standing, Gray's Anatomy, The Anatomical Basis of Medicine and surgery 39th Edition, 2005; Elsivier Churchill Livingstone, P 1003-1004.

[2] Tandler J (1913) Anatomie des Herzens. In: Handbuch der Anatomie des Menchen. Van Bardeleben K (ed.). G. Fischer, Jena 84-90.

[3] Silver MD, Lam JHC, Ranganathan N, Wigle ED. Morphology of the human tricuspid valve. Circulation. 1971;43: 333-348

[4] Nigri GR, Di Dio LJ, Baptista CA Papillary muscles and tendinous cords of the right ventricle of the human heart: Morphological characteristics; SurgRadiol Anat. 2001; 23(1): 45-49.

[5] Lam JHC, Ranganathan N, Nigle ED, Silver MD. Morphology of the human Mitral Valve; T Chordae Tendinae: A new classification; Circulation, 1970 March; Vol. XL1: 449-458.

[6] Skwarek M, Hreczecha J, Dudziak M, Jerzemowski J, Grzybiak M. The morphology and distribution of the tendinous chords and their relation to the papillary muscles in the tricuspid valve of the human hearts. Folia Morphol 2007; 66: 314-322.

[7] Mohamed A.B. Motabagani; Comparative Anatomical, Morphometric and Histological Studies of the Tricuspid Valve-Complex in Human and Some Mammalian Hearts. J.Anat.Soc. India 55 (1) 1-23 (2006).

[8] Escande G, Guillot M, Tanguy A, Vanneuvalle G. Anatomy of the right atrio ventricular valve. Description of a new type of Chordae: The mixed Chordae. Bull AssocAnat(Nancy) 1980 March; 64 (184): 73-82.

[9] Gunnal SA, Wabale RN, Farooqui MSMorphological study of chordae tendineae in human cadaveric hearts. Heart views. 2015;16(1): $1-12$

[10] Xanthos T, Dalivigkas I, EkmektzoglouKA.Anatomic variations of the cardiac valves and papillary muscles of the right heart. Ital J AnatEmbryol 2011; 116(2):111-26.

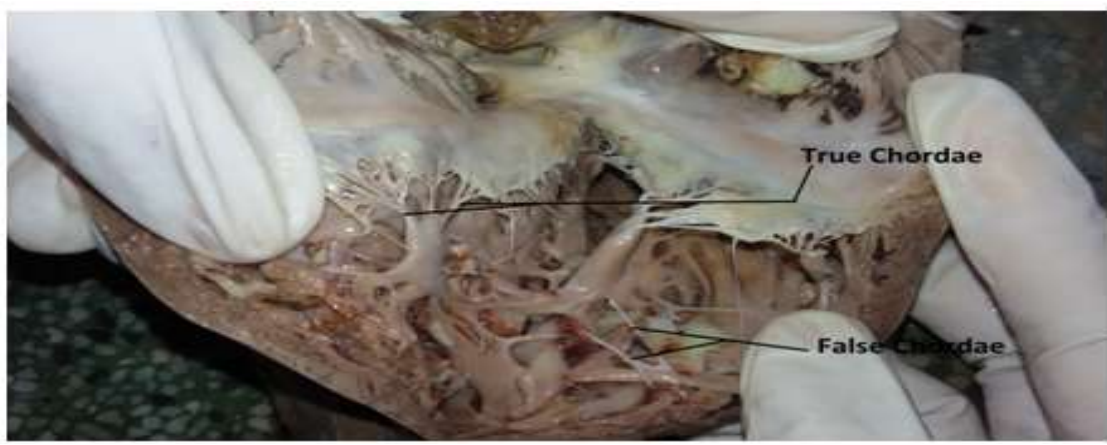

Photograph No. 1

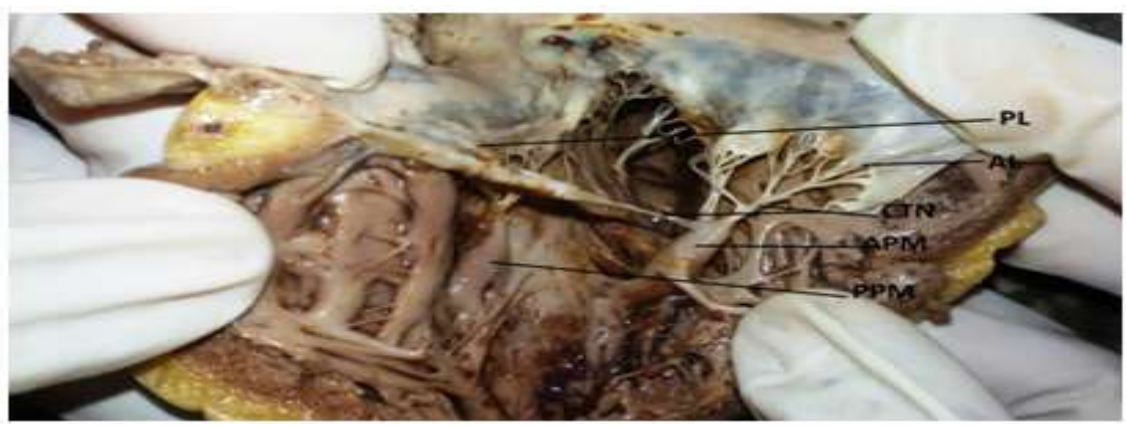

Photograph No. 2: Showing chordae tendineae arising from anterior papillary muscle is inserted into the corresponding leaflet (anterior leaflet) as well as the adjacent leaflet (posterior leaflet)

CTN -Chordae tendineae, APM - Anterior papillary muscle, PPM- Posterior papillary muscle, AL-Anterior Leaflet, PL - Posterior leaflet

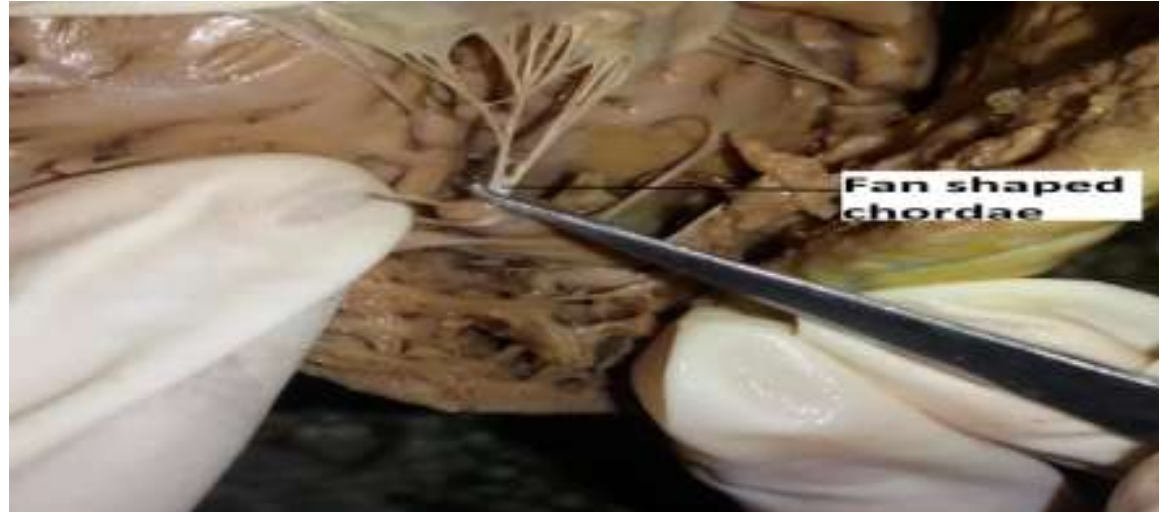

Photograph No. 3 